\title{
Icterícia neonatal e seus fatores perinatais associados: perfil dos recém-nascidos internados em uma unidade de terapia intensiva neonatal de maternidade de referência terciária no município de Fortaleza - Ceará
}

\section{Neonatal jaundice and its associated perinatal factors: profile of newborns hospitalized in a neonatal intensive care unit of a tertiary reference neonatal clinic in the municipatility of Fortaleza - Ceará}

\author{
Rafaela Loiola de Carvalho1 ${ }^{1}$. Maria Francielze Holanda Lavor ${ }^{1,2}$. \\ 1 Universidade Federal do Ceará (UFC), Maternidade Escola Assis Chateaubriand (MEAC), Fortaleza, Ceará, Brasil. 2 Instituto \\ Doutor José Frota, Fortaleza, Ceará, Brasil.
}

\section{RESUMO}

Objetivos: conhecer o perfil de recém-nascidos (RN) internados em unidade de terapia intensiva (UTIN), no Serviço de Neonatologia de uma maternidade pública terciária do município de Fortaleza, que apresentaram icterícia. Metodologia: estudo transversal, prospectivo, sendo os dados coletados nos prontuários de 01 de fevereiro a 31 de julho de 2018, utilizando questionário simples. As análises estatísticas foram realizadas no programa software R 3.3.1 e Microsoft Excel ${ }^{\circledR}$ 2016. Resultados: os dados revelaram que 70,7\% dos $\mathrm{RN}$ apresentaram icterícia significativa e 84,5\% tinham idade gestacional menor que 35 semanas. O sexo masculino apresentou-se em 58,2\% dos casos. Em $65,3 \%$ o tempo de clampeamento do cordão umbilical foi menor que 1 minuto. A taxa de sepse precoce foi de $68 \%$ e a de sepse tardia de $35,9 \%$. A hemorragia peri-intraventricular (HPIV) esteve presente em $37,4 \%$ da amostra. O índice de policitemia foi de 1\%. Pré-eclâmpsia foi fator protetor para icterícia significativa. Fototerapia foi realizada em todos os RN e 5,1\% necessitaram de exsanguíneotransfusão. Conclusões: a prematuridade abaixo de 35 semanas foi o principal fator associado a icterícia de alto risco e esforços obstétricos e neonatais voltados para a sua prevenção são necessários no sentido de melhorar esse quadro e seus fatores relacionados.

Palavras-chave: Icterícia neonatal. Unidades de Terapia Intensiva Neonatal. Fototerapia. Recém-nascido.

\section{ABSTRACT}

Objectives: the main objective was to know the profile of newborns (NB) hospitalized in a neonatal intensive care unit (NICU) in a tertiary reference Neonatal Clinic in the municipality of Fortaleza who presented neonatal jaundice. Methodology: it was a cross-sectional study, prospective, conducted during February-July 2018 through the use of a simple questionnaire. The data were analyzed with software R 3.3.1 e Microsoft Excel ${ }^{\circledR}$ 2016. Results: it was noted that 70,7\% of NB presented significant neonatal jaundice, $84,5 \%$ had gestational age less than 35 weeks. The majority of NB studied was male (58,2\%). Umbilical cord clamping time less than 1 minute represented $65,3 \%$ of cases studied. The rate of early and late sepsis was $68 \%$ e $35,9 \%$, respectively. Intracranial hemorrhage (IH) was associated to $37,4 \%$ of NB and polycythaemia to $1 \%$. Pre-eclampsia was protective factor for severe hyperbilirubinemia. All babies were submitted to phototherapy and 5,1\% needed exchange transfusion. Conclusions: the main factor of significant neonatal jaundice is premature birth under 35 weeks of gestacional age. Obstetrics and neonatal efforts are necessary to avoid it.

Keywords: Neonatal jaundice. Neonatal Intensive Care Units. Phototherapy. Newborn.

Autor correspondente: Rafaela Loiola de Carvalho, Rua Coronel Nunes de Melo, sem número, Rodolfo Teófilo, Fortaleza, Ceará. CEP: $60430-270$. Telefone: +5585 3366-8528. E-mail: carvrafaela@hotmail.com

Conflito de interesses: Não há qualquer conflito de interesses por parte de qualquer um dos autores.

Recebido em: 12 Fev 2019; Revisado em: 13 Jun 2019; Aceito em: 15 Jun 2019. 


\section{INTRODUÇÃO}

A icterícia é uma condição médica comum no período neonatal, sendo a principal causa de reinternação na primeira semana de vida. ${ }^{1}$ É considerada "fisiológica" quando ocorre após as primeiras $24 \mathrm{~h}$ de vida, sendo vista como patológica antes desse período ou quando permanece por períodos prolongados. Quando patológica e associada a altos níveis de bilirrubina, pode levar a um quadro grave de encefalopatia, o Kernicterus, ou até mesmo a óbito, se não for adequadamente tratada. ${ }^{2}$

Diversos fatores maternos e perinatais estão associados ao surgimento de icterícia neonatal. Dentre eles destacamse idade gestacional (IG), sexo do recém-nascido (RN), diabetes mellitus gestacional (DMG), pré-eclâmpsia, tempo de clampeamento do cordão umbilical, eliminação tardia de mecônio, nutrição enteral tardia e perda de peso importante na primeira semana de vida. Algumas situações geram o aumento da circulação êntero-hepática, como jejum prolongado e anomalias gastrointestinais (processos obstrutivos, estenose hipertrófica do piloro) e favorecem a hiperbilirrubinemia. Ainda se destacam como fatores importantes no surgimento dessa condição as doenças hemolíticas (incompatibilidades $\mathrm{ABO}$ e Rh) e a presença de coleções sanguíneas extravasculares (cefalohematoma, bossa serossanguínea e equimoses). ${ }^{3}$

O tratamento baseia-se em fototerapia e exsanguíneotransfusão, quando há predominância da bilirrubina indireta, sendo realizado a partir de dados retirados de gráficos e tabelas, que variam de acordo com a IG e a idade do RN. ${ }^{4}$

No contexto do presente estudo, é importante conhecer a classificação dos RN de acordo com a IG ao nascimento. A divisão apresenta-se da seguinte forma: pré-termos, prematuros extremos, muito prematuros, prematuros moderados e tardios, que correspondem a menores que 37 semanas, menores que 28 semanas, de 28 a 31 semanas e 6 dias, de 32 a 33 semanas e 6 dias e de 34 a 36 semanas e 6 dias, respectivamente. ${ }^{5}$

Diante do exposto, o estudo teve o objetivo de conhecer o perfil dos RN internados em unidade de terapia intensiva (UTIN) do Serviço de Neonatologia de uma maternidade pública terciária do município de Fortaleza-CE, no período de 01 de fevereiro a 31 de julho de 2018, que apresentaram icterícia.

\section{MATERIAL E MÉTODOS}

É um estudo transversal, prospectivo, onde os dados foram coletados nos prontuários, através de questionário simples. A população do estudo incluiu recém-nascidos internados na UTIN da Maternidade Escola Assis Chateaubriand (MEAC). Foram elegíveis todos os prontuários de neonatos que nasceram na MEAC e estiveram internados na UTIN no período de 01 de fevereiro a 31 de julho de 2018. Excluíram-se os pacientes que foram transferidos durante o processo de coleta de dados.

Para a captação dos dados, foi utilizada uma ficha de coleta, elaborada pela própria pesquisadora que continha variáveis relativas à mãe (tipo de parto, tipagem sanguínea, presença de DMG e de pré-eclâmpsia), ao RN (APGAR no primeiro minuto, sexo, idade gestacional, peso de nascimento, classificação do recém-nascido de acordo com a IG e com o peso de nascimento, tipagem sanguínea, coombs direto, tempo de eliminação de mecônio) e à assistência (tempo de clampeamento do cordão umbilical, tempo de jejum e de nutrição parenteral, perda de peso nas primeiras 72 horas de vida, valor de bilirrubina total (BT) antes de 24 horas de vida, entre 24 e 72 horas, entre 5 e 7 e após 7 dias de vida, necessidade de fototerapia e de exsanguíneotransfusão, tempo de internação em UTIN, desfecho final e presença de patologias como síndrome do desconforto respiratório (SDR), sepse precoce e tardia, cardiopatias, policitemia e hemorragia peri-intraventricular (HPIV), além de alterações como hipotermia e hipertermia e hematomas ou equimoses).

A IG em semanas foi subdividida nas seguintes categorias: $<28$, de 28 a 29, de 30 a 31, de 32 a 33, de 34 a menores que 35 e maiores ou iguais a 35 semanas. Foram selecionados como apresentando icterícia significativa, os RN que tinham valores de BT correspondentes aos pontos de corte para uso de fototerapia considerados de alto risco de acordo com a IG e a idade de nascimento, conforme dados presentes nos gráficos de Bhutani naqueles com IG maior ou igual a 35 semanas e nas tabelas da Academia Americana de Pediatria para IG menor que 35 semanas (TABELA 1). ${ }^{6,7}$

Tabela 1. Presença de icterícia significativa de acordo com valores de BT, IG e idade do RN. , $^{6}$

\begin{tabular}{lllll}
\hline Idade gestacional $^{*}$ & $\begin{array}{l}\text { BT com } \\
\text { menos de } \\
24 \text { horas }\end{array}$ & $\begin{array}{l}\text { BT de } \\
\text { 24 a 72 } \\
\text { horas }\end{array}$ & $\begin{array}{l}\text { BT de 5 a } \\
\text { 7 dias }\end{array}$ & $\begin{array}{l}\text { BT }>\text { ou=7 } \\
\text { dias }\end{array}$ \\
\hline$<28$ semanas & 5 & 5 & 5 & 5 \\
28 a 29 semanas & 6 & 6 & 6 & 6 \\
30 a 31 semanas & 8 & 8 & 8 & 8 \\
32 a 33 semanas & 10 & 10 & 10 & 10 \\
34 a <35 semanas & 12 & 12 & 12 & 12 \\
35-37 semanas & 8 & $8-13,5^{+}$ & 15 & 15 \\
$>$ ou=38 semanas & 10 & $10-15,5^{\S}$ & 18 & 18 \\
\hline
\end{tabular}

*Estratificação da IG de acordo com o gráfico de Bhutani para $\mathrm{IG}>\mathrm{ou}=35$ semanas e com a tabela da Academia Americana de Pediatria para $\mathrm{IG}<35$ semanas com delimitação dos valores de $\mathrm{BT}$ com pontos de corte para uso de fototerapia considerados de alto risco (correspondentes aos portadores de icterícia significativa).

+ BT com $24 \mathrm{~h}$ de vida: $>8 \mathrm{mg} / \mathrm{dL}$, BT com $36 \mathrm{~h}$ : >9,5mg/dL; BT com 48h: $>11,5 \mathrm{mg} / \mathrm{dL}$ e BT com $72 \mathrm{~h}:>13,5 \mathrm{mg} / \mathrm{dL}$

$\S \mathrm{BT}$ com $24 \mathrm{~h}$ de vida: $>10 \mathrm{mg} / \mathrm{dL}$, BT com $36 \mathrm{~h}:>11,5 \mathrm{mg} / \mathrm{dL}$; BT com 48h: $>13 \mathrm{mg} / \mathrm{dL}$ e BT com $72 \mathrm{~h}:>15,5 \mathrm{mg} / \mathrm{dL}$

$\mathrm{Na}$ análise das variáveis numéricas, os dados foram avaliados em médias e percentis. Nas variáveis categóricas, expostos em frequência e taxa de prevalência, de modo a investigar 
associações entre a icterícia neonatal e os seus fatores associados. Adotou-se um nível de significância de 5\%. Na investigação da associação entre as variáveis categóricas utilizou-se o teste de qui-quadrado de Pearson e o exato de Fisher. As análises estatísticas foram realizadas utilizando os programas estatísticos software R 3.3.1, JAMOVI 0.9.5.12 e o Microsoft Excel® 2016.

O presente estudo foi avaliado pelo Comitê de Ética em Pesquisa envolvendo seres humanos da Maternidade Escola da Universidade Federal do Ceará e aprovado através do parecer número 2.510.991.

\section{RESULTADOS}

Foram selecionados para o estudo 163 pacientes internados na UTIN da MEAC, sendo excluídos $23 \mathrm{RN}$, pois estes foram transferidos para outros hospitais. Dos 140 restantes, 70,7\% apresentaram icterícia significativa. Desses, 84,5\% tinham menos que 35 semanas, sendo $21,2 \%$ pré-termos extremos, $26,3 \%$ muito prematuros, $30,3 \%$ com prematuridade moderada e $13,1 \%$ tardia. Apenas $8,1 \%$ eram a termo (FIGURA 1 ). O sexo predominante foi o masculino $(58,2 \%)$, sendo $40 \% \mathrm{RN}$ do sexo feminino e $1,4 \%$ dos bebês com sexo indeterminado por genitália ambígua.

Figura 1. Estratificação dos pacientes estudados.

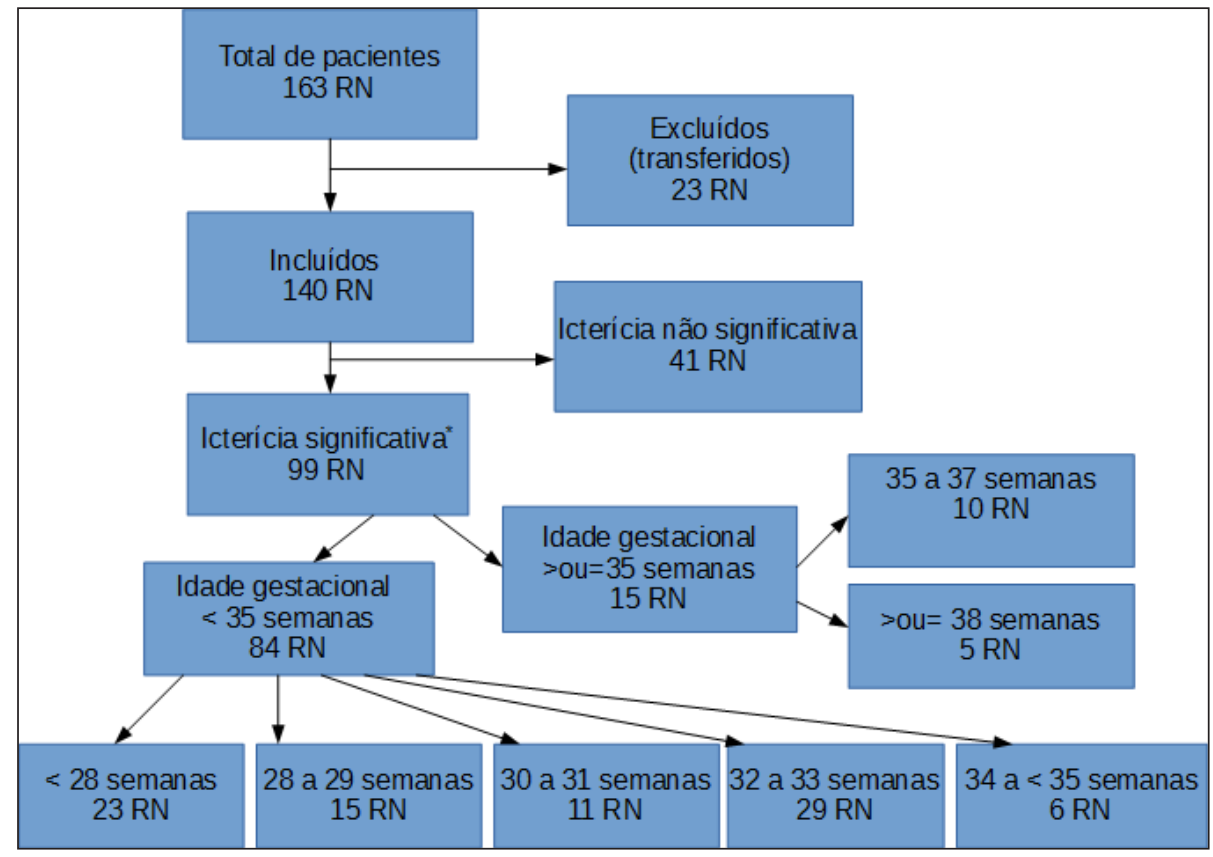

O peso do nascimento foi maior que 2500 gramas $(\mathrm{g})$ em $19,3 \%$ dos RN. Apresentaram peso abaixo de $2500 \mathrm{~g} 80,7 \%$ dos bebês, estando abaixo de $1500 \mathrm{~g}$ e de $1000 \mathrm{~g} 42,8 \%$ e $21,4 \%$, respectivamente.

A principal via de parto foi a cesárea, em $61,6 \%$ dos casos, por indicação materna ou fetal. Pré-eclâmpsia grave (PEG) e DMG, foram as principais causas maternas, com 31,1\% e $15,5 \%$ respectivamente. Relacionando o peso com a IG, $82,8 \%$ dos RN eram adequados para a idade gestacional (AIG). O escore APGAR no primeiro minuto de vida foi menor que 7 em $44,4 \%$ dos RN. O tempo de clampeamento do cordão umbilical foi menor que 1 minuto em $65,3 \%$, entre 1 e 3 minutos em $33,7 \%$ e maior que 3 minutos em $1 \%$ da amostra.

A tipagem sanguínea materna mais prevalente foi a $\mathrm{O}$ positivo (45,6\%), seguida da A positivo (35,9\%). Tipagens com Rh negativo somaram 5,8\% (A negativo 1,9\% e O negativo 3,9\%). Já em relação a tipagem dos $\mathrm{RN}$, a A positivo e a $\mathrm{O}$ positivo tiveram a mesma prevalência, representando $37,4 \%$ cada uma. O Coombs direto foi positivo em apenas $5,1 \%$ dos casos.
Das patologias apresentadas pelos RN durante a internação, destacaram-se a síndrome do desconforto respiratório (SDR), presente em $73,8 \%$, além da sepse precoce e tardia, com $68 \%$ e $35,9 \%$, respectivamente. Problemas cardíacos representaram $38,8 \%$ dos casos. A hipotermia foi outro fator de destaque nos pacientes estudados, tendo uma prevalência de $90,3 \%$, seguida da hipertermia, equivalente a $68 \%$ da amostra.

Dos RN em análise, a presença de hematomas ou equimoses ocorreu em apenas $10,1 \%$ dos casos e a hemorragia periintraventricular (HPIV) em 37,4\% da amostra. A policitemia apresentou-se somente em $1 \%$ dos pacientes.

A eliminação tardia de mecônio (após 48 horas de vida) ocorreu em $21,4 \%$ dos casos. O tempo de jejum por mais de 48 horas representou $26,2 \%$ da amostra, ficando $33 \%$ entre 24 e 48 horas e $40,8 \%$ com início da dieta nas primeiras 24 horas de vida. O início da nutrição parenteral precoce (menos de 24 horas de vida) aconteceu em $60,3 \%$ dos RN e o seu uso durou em média 14,3 dias. A perda de peso nas primeiras 72 horas após o nascimento foi menor que $7 \%$ em $46,5 \%$ dos pacientes e maior que $7 \%$ em 53,5\%. 
Quanto ao tratamento, 100\% apresentaram icterícia às custas de bilirrubina indireta e foram submetidos à fototerapia e 5,1\% realizaram exsanguíneotransfusão. Em 2,9\% dos casos, houve aumento importante da bilirrubina direta, necessitando de investigação adicional.
O tempo médio de internação na UTI neonatal foi de 21,3 dias. O desfecho dos bebês foi alta hospitalar em $87,9 \%$ dos casos e óbito em $12,1 \%$.

A TABELA 2 mostra os dados estatísticos comparativos da icterícia significativa com os fatores maternos e neonatais.

Tabela 2. Dados comparativos de icterícia significativa com as variáveis maternas e neonatais.

\begin{tabular}{|c|c|c|c|}
\hline Variáveis & $\begin{array}{c}\text { Icterícia significativa } \\
\mathbf{N}(\%)\end{array}$ & $\begin{array}{c}\text { Icteríca não significativa } \\
\text { N (\%) }\end{array}$ & p \\
\hline IG & & & $<0,012^{*}$ \\
\hline$>\mathrm{ou}=35 \mathrm{~s}$ & $16(15,5 \%)$ & $24(64,9 \%)$ & \\
\hline$<35 \mathrm{~s}$ & $87(84,5 \%)$ & $13(35,1 \%)$ & \\
\hline Sexo & & & $<0.638$ \\
\hline Feminino & $41(41,8 \%)$ & $15(37,5 \%)$ & \\
\hline Masculino & $57(58,2 \%)$ & $25(62,5 \%)$ & \\
\hline Tipo de parto & & & $<0.112$ \\
\hline Vaginal & $38(38,4 \%)$ & $10(24,4 \%)$ & \\
\hline Cesáreo & $61(61,6 \%)$ & $31(75,6 \%)$ & \\
\hline Peso ao nascimento & & & $<0.543$ \\
\hline$>2500 \mathrm{~g}$ & $16(16,2 \%)$ & $11(26,8 \%)$ & \\
\hline $2500-1500 \mathrm{~g}$ & $39(39,4 \%)$ & $14(34,1 \%)$ & \\
\hline $1500-1000 \mathrm{~g}$ & $23(23,2 \%)$ & $8(19,5 \%)$ & \\
\hline$<1000 \mathrm{~g}$ & $21(21,2 \%)$ & $8(19,5 \%)$ & \\
\hline Tempo de clampeamento do cordão umbilical & & & $<0.574$ \\
\hline$<1 \min$ & $64(65,3 \%)$ & $30(73,2 \%)$ & \\
\hline $1-3 \min$ & $33(33,7 \%)$ & $11(26,8 \%)$ & \\
\hline$>3 \mathrm{~min}$ & $1(1,0 \%)$ & $0(0 \%)$ & \\
\hline APGAR no primeiro minuto & & & $<0.837$ \\
\hline$<7$ & $44(44,4 \%)$ & $19(46,3 \%)$ & \\
\hline$>7$ & $55(55,6 \%)$ & $22(53,7 \%)$ & \\
\hline Peso de acordo com a IG & & & $<0.572$ \\
\hline AIG & $82(82,8 \%)$ & $30(75,0 \%)$ & \\
\hline PIG & $12(12,1 \%)$ & $7(17,5 \%)$ & \\
\hline GIG & $5(5,1 \%)$ & $3(7,5 \%)$ & \\
\hline $\mathrm{DMG}^{+}$ & $16(15,5 \%)$ & $9(24,3 \%)$ & $<0.316$ \\
\hline Pré-eclâmpsia & $32(31,1 \%)$ & $19(51,4 \%)$ & $<0.028^{*}$ \\
\hline Sepse Precoce & $70(68,0 \%)$ & $22(59,5 \%)$ & $<0.420$ \\
\hline Sepse tardia & $37(35,9 \%)$ & $14(37,8 \%)$ & $<0.844$ \\
\hline $\mathrm{SDR}^{\S}$ & $76(73,8 \%)$ & $27(73 \%)$ & $<0.923$ \\
\hline Cardiopatia & $40(38,8 \%)$ & $11(29,7 \%)$ & $<0.324$ \\
\hline Hipotermia & $93(90,3 \%)$ & $32(86,5 \%)$ & $<0.521$ \\
\hline Hipertermia & $70(68,0 \%)$ & $25(67,6 \%)$ & $<0.965$ \\
\hline Policitemia & $1(1,0 \%)$ & $0(0 \%)$ & $<0.518$ \\
\hline
\end{tabular}

Continua. 
Conclusão.

Tabela 2. Dados comparativos de icterícia significativa com as variáveis maternas e neonatais.

\begin{tabular}{|c|c|c|c|}
\hline Variáveis & $\begin{array}{c}\text { Icterícia significativa } \\
\mathbf{N}(\%)\end{array}$ & $\begin{array}{c}\text { Icteríca não significativa } \\
\mathbf{N}(\%)\end{array}$ & p \\
\hline Eliminação de mecônio & & & $<0.973$ \\
\hline$<48 \mathrm{~h}$ & $81(78,6 \%)$ & $29(78,4 \%)$ & \\
\hline$>48 \mathrm{~h}$ & $22(21,4 \%)$ & $8(21,6 \%)$ & \\
\hline Tempo de dieta zero & & & $<0.152$ \\
\hline$<24 \mathrm{~h}$ & $42(40,8 \%)$ & $14(37,8 \%)$ & \\
\hline $24-48 \mathrm{~h}$ & $34(33,0 \%)$ & $18(48,6 \%)$ & \\
\hline$>48 \mathrm{~h}$ & $27(26,2 \%)$ & $5(13,5 \%)$ & \\
\hline ABO-RH materno & & & $<0.543$ \\
\hline $\mathrm{O}+$ & $47(45,6 \%)$ & $22(59,5 \%)$ & \\
\hline O- & $4(3,9 \%)$ & $0(0 \%)$ & \\
\hline A- & $2(1,9 \%)$ & $1(2,7 \%)$ & \\
\hline $\mathrm{A}+$ & $37(35,9 \%)$ & $12(32,4 \%)$ & \\
\hline ABO-RH do RN & & & $<0.847$ \\
\hline $\mathrm{O}+$ & $37(37,4 \%)$ & $17(41,5 \%)$ & \\
\hline $\mathrm{A}+$ & $37(37,4 \%)$ & $17(41,5 \%)$ & \\
\hline A- & $1(1,0 \%)$ & $0(0 \%)$ & \\
\hline $\mathrm{B}+$ & $13(13,1 \%)$ & $3(7,3 \%)$ & \\
\hline B- & $1(1,0 \%)$ & $0(0 \%)$ & \\
\hline Coombs direto & $5(5,1 \%)$ & $0(0 \%)$ & $<0.824$ \\
\hline Duração da NPT ${ }^{* *}$ & & & $<0.372$ \\
\hline$<7$ dias & $49(81,7 \%)$ & $17(81 \%)$ & \\
\hline$>7$ dias & $13(20,6 \%)$ & $4(19 \%)$ & \\
\hline Perda Percentual de peso nas primeiras $72 \mathrm{~h}$ & & & $<0.438$ \\
\hline$<7 \%$ & $46(46,5 \%)$ & $22(53,7 \%)$ & \\
\hline$>7 \%$ & $53(53,5 \%)$ & $19(46,3 \%)$ & \\
\hline Hematomas e equimoses & $10(10,1 \%)$ & $3(7,3 \%)$ & $<0.606$ \\
\hline HPIV & $37(37,4 \%)$ & $16(37 \%)$ & $<0.855$ \\
\hline Fototerapia & $99(70,7 \%)$ & $41(28,3 \%)$ & - \\
\hline Exsanguíneotransfusão & $5(5,1 \%)$ & $0(0 \%)$ & $<0.143$ \\
\hline
\end{tabular}

* p estatisticamente significante; + Diabetes Mellitus Getacional; § Síndrome do Desconforto Respiratório; **Nutrição Parenteral.

\section{DISCUSSÃO}

A icterícia neonatal ocorre em $60 \%$ dos RN a termo e em $80 \%$ dos pré-termos. A maior frequência no prematuro decorre da imaturidade dos sistemas e do início da alimentação enteral não tão precocemente, o que aumenta a circulação ênterohepática. ${ }^{8,9}$ De acordo com a literatura, encontrou-se a maior prevalência da icterícia em pré-termos, $84,5 \%$ da amostra analisada, apresentando significância estatística $(p<0.012) \mathrm{e}$ com uma chance 2,95 vezes maior desses RN apresentarem essa doença ( $\mathrm{IC}=1.95-6.96)$. Estudo de Bhutani et al. encontrou resultado semelhante. ${ }^{10}$

O peso médio dos bebês foi de 1761g. Estudos em hospital terciário de Porto Alegre e em hospital de Tehran revelaram peso médio de $3054 \mathrm{~g}$ e de $1950 \mathrm{~g}$, respectivamente, em RN com icterícia significativa. ${ }^{11}$ Porém o peso ao nascimento não apresentou significância estatística $(p<0.543)$.

Comparando-se o sexo mais prevalente encontrado no estudo, o masculino $(58,2 \%)$, os resultados foram semelhantes ao de outras duas pesquisas, citadas acima $(52,7 \%$ em hospital terciário de Porto Alegre e 56,4\% em hospital de Tehran), porém estatisticamente sem significância $(p<0.638){ }^{11,12}$

O parto cesáreo representou a maior parte dos casos, o que concordou com estudo realizado em Porto Alegre, porém não se mostrou significante estatisticamente $(p<0.112) .{ }^{12} \mathrm{As}$ 
principais condições maternas foram PEG e DMG, fatores sabidamente associados a icterícia, porém, no presente estudo, a hipertensão arterial mostrou-se um fator protetor $(p<0.028)$, representando 2,3 vezes menos chances de desenvolver a doença $(\mathrm{IC}=0.198-0.920)$. Isso diverge do estudo de Marra et $a l$. em que a pré-eclâmpsia esteve associada a icterícia. ${ }^{13}$ Já a relação da icterícia neonatal com a DMG não se mostrou significativamente estatísca. ${ }^{7}$

Estudos mostram que o clampeamento tardio do cordão umbilical leva a maiores índices de icterícia neonatal. Pesquisa realizada em São Paulo, no Hospital Geral de Grajaú, mostrou que o clampeamento precoce foi fator protetor para icterícia, apresentando-se em 73,8\% dos RN que realizaram esse procedimento. ${ }^{14} \mathrm{O}$ presente estudo apresentou valor absoluto semelhante $(65,3 \%)$, porém sem significância clínica estatisticamente $(p<0.574)$, já que, mesmo com o cordão clampeado precocemente, os RN tiveram icterícia significativa. ${ }^{7}$

A tipagem sanguínea materna mais prevalente foi a $\mathrm{O}$ positivo, seguido pela A positivo, achados concordantes com dois estudos (Keren et al. e Enk et al.). Já quanto aos $\mathrm{RNs}$, as tipagens sanguíneas principais foram A positivo e $\mathrm{O}$ positivo com mesmo percentual $(37,4 \%)$. O Coombs direto (CD) foi positivo numa minoria dos casos $(5,1 \%)$, sendo todos associados a incompatibilidade ABO e com IG maior ou igual a 35 semanas. Maisels et al. encontraram 22\% dos pacientes com CD positivo, divergindo do achado do presente estudo, porém Enk et al. encontraram valor aproximado $(2,7 \%){ }^{13,15,16} \mathrm{~A}$ análise estatística não mostrou significância clínica $(p<0.824)$.

A sepse, fator associado à icterícia por alterar a capacidade de ligação da bilirrubina à albumina, se mostrou com alta prevalência na amostra analisada (68\% precoce e $35,9 \%$ tardia) porém sem apresentar significância estatística $(p<0.420$ para sepse precoce e $p<0.844$ para tardia), mostrando discordância com estudo realizado por Taheri et al., que só encontrou 11,7\% dos casos (soma total). ${ }^{6} 11$ A diferença provavelmente se deve ao fato de que no presente estudo foi considerada a sepse com diagnóstico clínico, enquanto Taheri et al. utilizaram apenas aquela amostra com hemocultura positiva.

A presença de equimoses e hematomas também foram comparadas com icterícia significativa, já que também são fatores sabidamente relacionados ao alto risco da doença, mas não apesentaram significância estatística, com $p<0.606$ e $p<0.855$, respectivamente, diferente do que há descrito na literatura. ${ }^{3}$

A eliminação precoce de mecônio (com menos de 48 horas de vida) está associada a menores níveis de bilirrubina. Esse achado foi o mais encontrado nos $\mathrm{RN}$ do presente estudo, porém estes apresentaram icterícia significativa, o que discorda da literatura. $\mathrm{O}$ achado não apresentou relevância estatística. ${ }^{17}$

Outros aspectos demonstrados foram o início tardio da nutrição enteral (após 24 horas de vida) e o uso prolongado de nutrição parenteral associados a altos níveis de bilirrubina, sendo estes dados concordantes com os encontrados na literatura. Todos os achados não foram estatisticamente significantes. ${ }^{17}$

O percentual de perda de peso maior ou igual a $7 \%$, fator de risco sabidamente associado a hiperbilirrubinemia, foi praticamente semelhante ao menor que $7 \%$, o que estatisticamente não demonstrou significância clínica $(p<0.438){ }^{2}$

Todos os RN foram submetidos a tratamento com fototerapia com o objetivo de evitar a encefalopatia bilirrubínica, forma grave da doença, e um pequeno percentual realizou exsanguíneotransfusão $(5,1 \%)$, sendo exatamente aqueles que tinham $\mathrm{CD}$ positivo e incompatibilidade $\mathrm{ABO}{ }^{2}$ Enk et al encontraram uma taxa de fototerapia de $97,3 \%$ e de exsanguíneotransfusão de $1,4 \%$, em concordância com os achados do presente estudo. ${ }^{12}$

O tempo de internação hospitalar e o seu desfecho foram influenciados por outras enfermidades além da icterícia, não apresentando esses dados significância estatística.

$\mathrm{O}$ estudo demonstrou que os prematuros menores que 35 semanas têm maior risco de desenvolver icterícia significativa, o que corrobora com a literatura. Outro achado com significância estatística, porém divergente do que já existe descrito, foi a hipertensão materna como fator protetor. Os demais fatores perinatais associados, conhecidos influenciadores da hiperbilirrubinemia, não se mostraram relevantes nos achados aqui presentes. Conclui-se que a prematuridade abaixo de 35 semanas é o principal fator associado a icterícia de alto risco e esforços obstétricos e neonatais voltados para a sua prevenção são necessários no sentido de melhorar esse quadro e seus fatores relacionados.

\section{REFERÊNCIAS}

1. Canadian Paediatric Society. Guidelines for detection, management and prevention of hyperbilirubinemia in term and late preterm newborn infants (35 or more week's gestation) - summary. Paediatr Child Health. 2007;12(5):401-18.

2. Bhutani VK, Johnson L. Prevenção de hiperbilirrubinemia neonatal grave em lactentes saudáveis com 35 ou mais semanas de gestação: implante de uma abordagem sistemática. J Pediatr. 2007;83(4):289-93.

3. Araújo BF. Manejo da icterícia no recém-nascido. In: Ferreira JP, organizador. Pediatria: diagnóstico e tratamento. Porto Alegre: Artmed; 2005. p.39-45.

4. Procianoy RS, Silveira RC, Pereira MR, Ribeiro MA, Grossi SP, Boher MA, et al. Problemas comuns no alojamento conjunto. In: Nader SS, Pereira DN. Atenção integral ao recém-nascido: guia de supervisão de saúde. Porto Alegre: Artmed; 2004. p.105-9.

5. American Academy of Pediatrics; The American College of Obstetricians and Gynecologists. Guidelines for Perinatal Care. 8. ed. Itasca: American Academy of Pediatrics; Washington: The American College of Obstetricians and Gynecologists; 2017.

6. Bhutani VK, Johnson L, Sivieri EM. Predictive ability of a presdicharge hour-specific serum bilirrubin for subsquent significant 
hyperbilirrubinemia in health term and near-term newborn. Pediatrics. 1999;103(1):6-14.

7. American Academy of Pediatrics. Management of hyperbilirrubinemia in the newborn infant 35 or more weeks of gestation. Pediatrics. 2004;114(1):297-316.

8. Watchko JF, Maisels MJ. Jaundice in low birthweight infants: pathobiology and outcome. Arch Dis Child Fetal Neonatal Ed. 2003;88(6):455-8.

9. Almeida MF, Nader PJ, Draque CM. Icterícia neonatal. In: Lopez FA, Campos D Jr, editores. Tratado de Pediatria. 2. ed. São Paulo: Manole; 2010. p. 1515-26.

10. Bhutani VK, Stark AR, Lazzeroni LC, Poland R, Gourley GR, Kazmierczak S, et al. Predischarge screening for severe neonatal hyperbilirubinemia identifies infants who need phototherapy. J Pediatr. 2013;162(3):477-82.

11. Taheri PA, Sadeghi M, Sajjadian N. Severe neonatal hyperbilirubinemia leading to exchange transfusion. Med J Islam Repub Iran. 2014;28(1):64.

12. Enk I, Abegg MP, Alves RJ, Stringhani F, Campos JF, Menezes
HS, et al. Icterícia como causa de internação neonatal: a experiência em um serviço terciário de Porto Alegre, RS. Revista da AMRIGS. 2009;53(4):361-7.

13. Marra NB, Nascimento DW, Sousa FL, Paltronieri MR, Guidoni RG, Toledo SF, et al. Prematuridade eletiva e as suas repercussões perinatais nas síndromes hipertensivas da gestação. UNILUS Ensino Pesqui. 2016;13(32):26-32.

14. Sarli YO. Associação entre tempo de clampeamento do cordão umbilical e icterícia neonatal precoce em recém-nascidos a termo [Internet]. São Paulo: Universidade Santo Amaro; 2018 [acesso em: 15 jan 2019]. Disponível em: http://dspace.unisa.br/ handle/123456789/183

15. Keren R, Luan X, Friedman S, Saddlemire S, Cnaan A, Bhutani VK. A comparison of alternative risk-assessment strategies for predicting significant neonatal hyperbilirrubinemia in term and nearterm infants. Pediatrics. 2008;121(1):170-9.

16. Maisels MJ, Kring E. Rebound in serum bilirubin level following intensive phototherapy. Arch Pediatr Adolesc Med. 2002;156(7):669-72.

17. Bertini G, Dani C, Pezzatti M, Rubaltelli FF. Prevention of bilirubin encephalopathy. Biol Neonate. 2001;79(3-4):219-23.

\section{Como citar:}

Carvalho RL, Lavor MF. Icterícia neonatal e seus fatores perinatais associados: perfil dos recém-nascidos internados em uma unidade de terapia intensiva neonatal de maternidade de referência terciária no município de Fortaleza - Ceará. Rev Med UFC. 2020 abr-jun;60(2):11-17. 